\title{
Uniqueness of invariant Lagrangian graphs in a homology or a cohomology class
}

\author{
Albert Fathi, Alessandro Giuliani And Alfonso Sorrentino
}

\begin{abstract}
Given a smooth compact Riemannian manifold $M$ and a Hamiltonian $H$ on the cotangent space $\mathrm{T}^{*} M$, strictly convex and superlinear in the momentum variables, we prove uniqueness of certain "ergodic" invariant Lagrangian graphs within a given homology or cohomology class. In particular, in the context of quasi-integrable Hamiltonian systems, our result implies global uniqueness of Lagrangian KAM tori with rotation vector $\rho$. This result extends generically to the $C^{0}$-closure of KAM tori.
\end{abstract}

Mathematics Subject Classification (2000): $37 \mathrm{~J} 50$ (primary); 37J40, 53D12 (secondary).

\section{Introduction}

A particularly interesting and fruitful approach to the study of local and global properties of dynamical systems is concerned with the study of invariant submanifolds, rather than single orbits, paying particular attention to their existence, their "fate" and their geometric properties. In the context of quasi-integrable Hamiltonian systems, one of the most celebrated breakthroughs in this kind of approach was KAM theory, which provided a method to construct invariant submanifolds diffeomorphic to tori, on which the dynamics is conjugated to a quasi-periodic motion with rotation vector $\rho$, sometimes referred to as KAM tori. KAM theory finally settled the old question about existence of such invariant submanifolds in "generic" quasiintegrable Hamiltonian systems, dating back at least to Poincaré, and opened the way to a new understanding of the nature of Hamiltonian systems, of their stability and of the onset of chaos in classical mechanics. However, the natural question about the uniqueness of these invariant submanifolds for a fixed rotation vector $\rho$ remained open for many more years and, quite surprisingly, even nowadays, for many respects it is still unanswered. A possible reason for this is that the analytic methods, which the KAM algorithm is based on, are not well suited for studying global questions, while, on the other hand, the natural variational methods to ap- 
proach this problem have been developed only much more recently and they are still not so widely well-known.

In this paper we address the above problem and prove global uniqueness of certain "ergodic" invariant Lagrangian graphs within a given homology or cohomology class, for a large class of convex Hamiltonians, known as Tonelli Hamiltonians. Our work will be based on the variational approach provided by the so-called Aubry-Mather theory, as well as its functional counterpart called weak KAM theory.

The paper is organized as follows. In Section 2 we define the geometric objects we shall look at (the invariant Lagrangian graphs), we introduce some concepts (homology class of an invariant measure and Schwartzman ergodicity), which will turn out to be useful for illustrating their dynamical properties, and we state our main uniqueness results. In Section 3 we prove our main results. In Section 4 we discuss the implications of our results for KAM theory and compare them with some previous local uniqueness theorems for KAM tori. In Appendix A we shall discuss some details concerning the definition of Schwartzman ergodicity, give some examples and describe some properties of Schwartzman ergodic flows.

ACKNOWLEDGEMENTS. We would like to thank Giovanni Gallavotti for having drawn our attention to this problem and for useful discussions. Albert Fathi and Alfonso Sorrentino are grateful to John Mather for many fruitful conversations.

\section{Setting and main results}

Let $M$ be a compact and connected smooth manifold without boundary of dimension $n$. Denote by TM its tangent bundle and $\mathrm{T}^{*} M$ the cotangent one. A point of T $M$ will be denoted by $(x, v)$, where $x \in M$ and $v \in \mathrm{T}_{x} M$, and a point of $\mathrm{T}^{*} M$ by $(x, p)$, where $p \in \mathrm{T}_{x}^{*} M$ is a linear form on the vector space $\mathrm{T}_{x} M$. Let us fix a Riemannian metric $g$ on $M$ and let $\|\cdot\|_{x}$ be the norm induced by $g$ on $\mathrm{T}_{x} M$; we shall use the same notation for the norm induced on the cotangent space $\mathrm{T}_{x}^{*} M$. This cotangent space $\mathrm{T}^{*} M$ can be canonically endowed with a symplectic structure, given by the exact 2-form $\omega=d x \wedge d p=-d(p d x)$, where $(\mathcal{U}, x)$ is a local coordinate chart for $M$ and $\left(\mathrm{T}^{*} \mathcal{U}, x, p\right)$ the associated cotangent coordinates. The 1 -form $\lambda=p d x$ is also called tautological form (or Liouville form) and is intrisically defined, i.e. independently of the choice of local coordinates. A distinguished role in the study of the geometry of a symplectic space is played by the so-called Lagrangian submanifolds.

Definition 2.1. Let $\Lambda$ be an $n$-dimensional $C^{1}$ submanifold of $\left(\mathrm{T}^{*} M, \omega\right)$. We say that $\Lambda$ is Lagrangian if for any $(x, p) \in \Lambda, \mathrm{T}_{(x, p)} \Lambda$ is a Lagrangian subspace, i.e. $\left.\omega\right|_{\mathrm{T}_{(x, p)} \Lambda}=0$.

We shall mainly be concerned with Lagrangian graphs, that is Lagrangian manifolds $\Lambda \subset T^{*} M$ such that $\Lambda=\{(x, \eta(x)), x \in M\}$. It is straightforward to check 
that the graph $\Lambda$ is Lagrangian if and only if $\eta$ is a closed 1-form. The element $c=[\eta] \in \mathrm{H}^{1}(M ; \mathbb{R})$ is called the cohomology class, or Liouville class, of $\Lambda$. This motivates the following extension of the notion of Lagrangian graph to the Lipschitz case.

Definition 2.2. A Lipschitz section $\Lambda$ of $\mathrm{T}^{*} M$ is a Lipschitz Lagrangian graph if it locally coincides with the graph of an exact differential.

Observe that a Lipschitz Lagrangian graph $\Lambda$ is differentiable almost everywhere and at each differentiability point $(x, p)$ the tangent space $\mathrm{T}_{(x, p)} \Lambda$ is a Lagrangian subspace. Such Lipschitz graphs, although less regular, enjoy many properties of $C^{1}$-Lagrangian graphs (see for instance [26]). In the following, when referring to a Lagrangian graph without specifying its regularity, we shall assume that it is at least Lipschitz.

We shall consider the dynamics on $\mathrm{T}^{*} M$ generated by a Tonelli Hamiltonian.

Definition 2.3. A function $H: \mathrm{T}^{*} M \longrightarrow \mathbb{R}$ is called a Tonelli (or optical) Hamiltonian if:

i) the Hamiltonian $H$ is of class $C^{k}$, with $k \geq 2$;

ii) the Hamiltonian $H$ is strictly convex in the fiber in the $C^{2}$ sense, i.e. the second partial vertical derivative $\partial^{2} H / \partial p^{2}(x, p)$ is positive definite, as a quadratic form, for any $(x, p) \in \mathrm{T}^{*} M$;

iii) the Hamiltonian $H$ is superlinear in each fiber, i.e.

$$
\lim _{\|p\|_{x \rightarrow+\infty}} \frac{H(x, p)}{\|p\|_{x}}=+\infty
$$

(because of the compactness of $M$, this condition is independent of the choice of the Riemannian metric).

Given $H$, we can define the associated Lagrangian, as a function on the tangent bundle:

$$
\begin{aligned}
L: \mathrm{T} M & \longrightarrow \mathbb{R} \\
(x, v) & \longmapsto \sup _{p \in \mathrm{T}_{x}^{*} M}\left\{\langle p, v\rangle_{x}-H(x, p)\right\}
\end{aligned}
$$

where $\langle\cdot, \cdot\rangle_{x}$ represents the canonical pairing between the tangent and cotangent space. If $H$ is a Tonelli Hamiltonian, one can easily prove that $L$ is finite everywhere, of class $C^{k}$, superlinear and strictly convex in the fiber in the $C^{2}$ sense (i.e. $L$ is a Tonelli Lagrangian) and the associated Euler-Lagrange flow $\Phi_{t}^{L}$ of $L$ is conjugated to the Hamiltonian flow $\Phi_{t}^{H}$ of $H$ via the Legendre transform:

$$
\begin{aligned}
\mathcal{L}: \mathrm{T} M & \longrightarrow \mathrm{T}^{*} M \\
(x, v) & \longmapsto\left(x, \frac{\partial L}{\partial v}(x, v)\right) .
\end{aligned}
$$


From now on we shall fix $H$ and denote by $L$ its conjugated Lagrangian and, when referring to an "invariant" measure or set, we shall understand "invariant with respect to the Hamiltonian flow generated by $H$ " or "with respect to the EulerLagrange flow generated by $L "$.

Given an invariant probability measure $\mu$ on TM one can associate to it an element $\rho(\mu)$ of the homology group $\mathrm{H}_{1}(M ; \mathbb{R})$, known as rotation vector or Schwartzman asymptotic cycle, which generalizes the notion of rotation vector given by Poincaré and describes how, asymptotically, a $\mu$-average orbit winds around TM. In fact, it is easy to show [20] that since $\mu$ is invariant by the Euler-Lagrangian flow $\Phi_{t}^{L}$, if $\eta=d f$ is an exact form, then $\int\langle d f, v\rangle d \mu=0$. Therefore, one can define a linear functional

$$
\begin{aligned}
\mathrm{H}^{1}(M ; \mathbb{R}) & \longrightarrow \mathbb{R} \\
c & \longmapsto \int_{\mathrm{T} M}\langle\eta, v\rangle d \mu,
\end{aligned}
$$

where $\eta$ is any closed 1 -form on $M$, whose cohomology class is $c$. By duality, there exists $\rho(\mu) \in \mathrm{H}_{1}(M ; \mathbb{R})$ such that

$$
\int_{\mathrm{T} M}\langle\eta, v\rangle d \mu=\langle c, \rho(\mu)\rangle \quad \forall c \in \mathrm{H}^{1}(M ; \mathbb{R}) .
$$

$\rho(\mu)$ is what we call the rotation vector of $\mu$ and it coincides with the Schwartzman asymptotic cycle of $\mu$. See Appendix A and [20] for more details.

This allows us to define the homology class of certain invariant Lagrangian graphs.

Definition 2.4. A Lagrangian graph $\Lambda$ is called Schwartzman uniquely ergodic if all invariant measures supported on $\Lambda$ have the same rotation vector $\rho$, which will be called homology class of $\Lambda$. Moreover, if there exists an invariant measure with full support, $\Lambda$ will be called Schwarztman strictly ergodic.

We are now ready to state our main result.

Main result. For any given $\rho \in \mathrm{H}_{1}(M ; \mathbb{R})$, there exists at most one Schwarzman strictly ergodic invariant Lagrangian graph with homology class $\rho$. [Theorem 3.4, Section 3.]

For sake of completeness, we also recall the following well-known result, which is a corollary of the results in [20] (see Section 3 below for a proof).

Well-known result. For any given $c \in \mathrm{H}^{1}(M ; \mathbb{R})$, there exists at most one invariant Lagrangian graph $\Lambda$ with cohomology class $c$, carrying an invariant measure whose support is the whole of $\Lambda$. [Theorem 3.2, Section 3.]

If $M=\mathbb{T}^{n}$, it is natural to ask for the implications of our result for KAM theory. In this case, the homology group $\mathrm{H}_{1}\left(\mathbb{T}^{n} ; \mathbb{R}\right)$ is canonically identified with $\mathbb{R}^{n}$, and the invariant manifolds of interest are the so-called KAM tori, defined as follows. 
Definition 2.5. $\mathcal{T} \subset \mathbb{T}^{n} \times \mathbb{R}^{n}$ is a (maximal) KAM torus with rotation vector $\rho$ if:

i) $\mathcal{T} \subset \mathbb{T}^{n} \times \mathbb{R}^{n}$ is a Lipschitz graph over $\mathbb{T}^{n}$;

ii) $\mathcal{T}$ is invariant under the Hamiltonian flow $\Phi_{t}^{H}$ generated by $H$;

iii) the Hamiltonian flow on $\mathcal{T}$ is conjugated to a uniform rotation on $\mathbb{T}^{n}$; i.e. there exists a diffeomorphism $\varphi: \mathbb{T}^{n} \rightarrow \mathcal{T}$ such that $\varphi^{-1} \circ \Phi_{t}^{H} \circ \varphi=R_{\rho}^{t}, \forall t \in \mathbb{R}$, where $R_{\rho}^{t}: x \mapsto x+\rho t$.

The celebrated KAM Theorem, whose statement will be recalled in Section 4, gives sufficient conditions on $H$ and on the rotation vector $\rho$, allowing one to construct a KAM torus with rotation vector $\rho$ and prescribed regularity (depending on the regularity class of $H$ ). Its proof is constructive and the invariant torus one manages to construct is locally unique (in a sense that will be clarified in Section 4). In spite of the long history and the huge literature dedicated to the KAM theorem, the issue of global uniqueness of such tori is still object of some debate and study, see for instance [5]. Our main result settles such question in the case of Tonelli Hamiltonians.

Corollary 2.6 (Global uniqueness of KAM tori). Every Tonelli Hamiltonian $H$ on $\mathrm{T}^{*} \mathbb{T}^{n}$ possesses at most one Lagrangian KAM torus for any given rotation vector $\rho$. In particular, if $H$ and $\rho$ satisfy the assumptions of the KAM Theorem, then there exists one and only one KAM torus with rotation vector $\rho$.

The property of being Lagrangian plays a crucial role. As it was observed by Herman [15], when $\rho$ is rationally independent, i.e. $\langle\rho, v\rangle \neq 0, \forall v \in \mathbb{Z}^{n} \backslash\{0\}$, as assumed in the KAM theorem, every KAM torus with frequency $\rho$ is automatically Lagrangian. On the other hand, the existence of Lagrangian KAM tori with rationally dependent frequency is not typical. In some cases, a variant of the classical KAM algorithm allows one to construct resonant invariant tori with a given rational rotation vector $\rho$, also known as lower dimensional tori $[13,14]$. However, typically they do not foliate any Lagrangian submanifold. Therefore, the question of uniqueness of resonant tori is more subtle and, to our knowledge, apart from a few partial results [7], it is still open.

In Section 4 we shall extend Corollary 2.6 to generic invariant tori contained in the $C^{0}$-closure of the set of KAM tori. As remarked by Herman [16], generically this set is much larger than the set of KAM tori, and typically the flow on such invariant manifolds is not conjugated to a rotation. See Section 4 for a more detailed discussion of these issues.

\section{Minimizing measures and Lagrangian graphs}

In this section we shall prove the main results announced in Section 2. Our proof will be based on Mather's variational approach to the study of Lagrangian systems, which is concerned with the study of action minimizing invariant probability measures (also called Mather's measures) and action minimizing orbits of the EulerLagrange flow. In particular, the keystone of such an approach consists in studying 
a family of modified Tonelli Lagrangians given by $L_{\eta}(x, v)=L(x, v)-\langle\eta(x), v\rangle$, where $\eta$ is a closed 1-form on $M$. These Lagrangians, in fact, have all the same Euler-Lagrange flow as $L$, but different action minimizing orbits/measures, according to the cohomology class of $\eta$. In this way, Mather defined for each cohomology class $c \in \mathrm{H}^{1}(M ; \mathbb{R})$ two compact invariant subsets of $\mathrm{T} M$ :

- $\widetilde{\mathcal{M}}_{c}$, the Mather set of cohomology class $c$, given by the union of the supports of all invariant probability measures that minimize the action of $L_{\eta}$ (c-action minizimizing measures or Mather's measures of cohomology class $c$ ), where $\eta$ is any closed 1-form on $M$ with cohomology class $[\eta]=c$;

- $\widetilde{\mathcal{A}}_{c}$, the Aubry set of cohomology class $c$, given by the union of all regular global minimizers of the action of $L_{\eta}$ (or $c$-regular minimizers); see [10,21] for a precise definition. It is convenient to recall here that, if: (i) $\alpha(c)$ is Mather's $\alpha$-function, i.e., the maximum over the invariant probability measures $\mu$ of $-\int L_{\eta} d \mu$, with $[\eta]=c$, and (ii) $\mathcal{S}_{\eta}$ is the set of critical subsolutions of $H_{\eta}(x, p):=H(x, \eta+p)$, i.e., the set of locally Lipschitz functions $u: M \longrightarrow$ $\mathbb{R}$ such that $H_{\eta}\left(x, d_{x} u\right) \leq \alpha(c)$ for almost every $x \in M$, then $\widetilde{\mathcal{A}}_{c}=\mathcal{L}^{-1}\left(\mathcal{A}_{c}^{*}\right)$, with

$$
\mathcal{A}_{c}^{*}=\bigcap_{u \in \mathcal{S}_{\eta}}\left\{\left(x, \eta_{x}+d_{x} u\right): u \text { is differentiable at } x\right\} \subset \mathrm{T}^{*} M,
$$

see $[10,12]$.

One can show that $\widetilde{\mathcal{M}}_{c} \subseteq \widetilde{\mathcal{A}}_{c}$ and, as proved by Carneiro in [6], that they are both contained in the energy level $\tilde{\mathcal{E}}_{c}=\{(x, v) \in \mathrm{T} M: H \circ \mathcal{L}(x, v)=\alpha(c)\}$. Moreover, one of the most important features of these sets is that they are graphs over $M$ (Mather's graph theorem [20]); namely, if $\pi:$ T $M \rightarrow M$ denotes the canonical projection, then $\pi \mid \widetilde{\mathcal{A}}_{c}$ is injective and its inverse $\left(\pi \mid \widetilde{\mathcal{A}}_{c}\right)^{-1}: \mathcal{A}_{c} \longrightarrow \widetilde{\mathcal{A}}_{c}$ is Lipschitz. This is the multidimensional analogue of Birkhoff's theorem for twist maps [4].

Analogously, for any rotation vector $h \in \mathrm{H}_{1}(M ; \mathbb{R})$, one can define another compact invariant subset of TM:

- $\widetilde{\mathcal{M}}^{h}$, the Mather set of homology class $h$, given by the union of the supports of all invariant probability measures with rotation vector $h$ that minimize the action of L (Mather's measures of homology class $h$ ).

One can show that $\widetilde{\mathcal{M}}^{h}$ also enjoys the graph property. See Lemma 3.5 for the relation between $\widetilde{\mathcal{M}}^{h}$ and $\widetilde{\mathcal{M}}_{c}$. For more details on Mather's theory for Lagrangian systems, we refer the reader to $[8,10,20]$.

Let us start now by proving some action minimizing properties of probability measures supported on Lagrangian graphs.

Given a Lagrangian graph $\Lambda$ with Liouville class $c$, we shall say that $\Lambda$ is $c$ subcritical, or simply subcritical, if $\Lambda \subset\left\{(x, p) \in \mathrm{T}^{*} M: H(x, p) \leq \alpha(c)\right\}$, 
where $\alpha$ is Mather's $\alpha$-function (see the lines preceding Equation (3.1) for a definition). The interest in such graphs comes from the fact that this is the smallest energy sub-level of $H$, containing Lagrangian graphs of cohomology $c$ (see [10]).

Given a subcritical Lagrangian graph $\Lambda$ with Liouville class $c$, we shall call $\Lambda_{\text {crit }}=\{(x, p) \in \Lambda: H(x, p)=\alpha(c)\}$ its critical part. The key result we need to prove is the following characterization of minimizing measures.

Lemma 3.1. Let $\mu$ be an invariant probability measure on $\mathrm{T} M$ and $\mu^{*}=\mathcal{L}_{*} \mu$ its push-forward to $\mathrm{T}^{*} M$, via the Legendre transform $\mathcal{L}$. Then, $\mu$ is a Mather's measure if and only if supp $\mu^{*}$ is contained in the critical part of a subcritical Lagrangian graph. In particular, any invariant probability measure $\mu^{*}$ on $\mathrm{T}^{*} M$, whose support is contained in an invariant Lagrangian graph with Liouville class $c$, is the image, via the Legendre transform, of a c-action minimizing measure.

Proof. (i) If $\mu$ is a Mather measure of cohomology class $c$, then the support of $\mu^{*}$ is contained in $\mathcal{L}\left(\widetilde{\mathcal{M}}_{c}\right) \subseteq \mathcal{L}\left(\widetilde{\mathcal{A}}_{c}\right)$. Since $\mathcal{L}\left(\widetilde{\mathcal{A}}_{c}\right)$ can be obtained as the intersection of all $c$-subcritical Lagrangian graphs $[3,10]$, then supp $\mu^{*}$ is contained in at least one $c$-subcritical Lagrangian graph $\Lambda$. In particular $\operatorname{supp} \mu^{*}$ is contained in the critical part of $\Lambda$, simply because, as recalled above, $\mathcal{L}\left(\widetilde{\mathcal{M}}_{c}\right)$ is contained in the energy level $\mathcal{E}_{c}^{*}=\left\{(x, p) \in \mathrm{T}^{*} M: H(x, p)=\alpha(c)\right\}$.

(ii) Let us fix $\eta$ to be a smooth closed 1-form with $[\eta]=c$, and let us assume that $\operatorname{supp} \mu^{*}$ is contained in the critical part of the $c$-subcritical Lagrangian graph $\Lambda=\{(x, \eta(x)+d u(x)), x \in M\}$, where $u: M \rightarrow \mathbb{R}$ is $C^{1,1}$. In order to show that $\mu$ is a $c$-action minimizing measure, it is enough to show that any orbit $\gamma$ in supp $\mu$ is a $c$-minimizer (i.e., for every finite time-interval $[a, b],\{\gamma(t)\}_{t \in[a, b]}$ minimizes the action of $L_{\eta},[\eta]=c$, among the curves with the same end-points $\gamma(a)$ and $\gamma(b))$, see $[10,19]$.

To this purpose, let us consider $(x, v) \in \operatorname{supp} \mu$ and let $\gamma(t) \equiv \pi\left(\Phi_{t}(x, v)\right)$, where $\Phi_{t}$ is the Euler-Lagrange flow and $\pi$ the canonical projection on $M$. Given any interval $[a, b] \subset \mathbb{R}$, let us consider the difference $u(\gamma(b))-u(\gamma(a))$ and rewrite it as:

$$
\begin{aligned}
u(\gamma(b))-u(\gamma(a)) & =\int_{a}^{b} d_{\gamma(s)} u(\gamma(s)) \dot{\gamma}(s) d s \\
& =\int_{a}^{b}\left[L_{\eta}(\gamma(s), \dot{\gamma}(s))+H_{\eta}\left(\gamma(s), d_{\gamma(s)} u\right)\right] d s
\end{aligned}
$$

where the second equality follows from the definition of the Hamiltonian as the Legendre-Fenchel transform of the Lagrangian and the fact that $\gamma(s)$ is an orbit of the Euler-Lagrange flow. Note that along the orbit $H_{\eta}\left(\gamma(s), d_{\gamma(s)} u\right)=\alpha(c)$, because supp $\mu$ is invariant and supp $\mu^{*}$ is in the critical part of $\Lambda$. Then

$$
\int_{a}^{b} L_{\eta}(\gamma(s), \dot{\gamma}(s)) d s=u(\gamma(b))-u(\gamma(a))-\alpha(c)(b-a) .
$$


On the other hand, any other curve $\gamma_{1}:[a, b] \rightarrow M$ such that $\gamma_{1}(a)=\gamma(a)$ and $\gamma_{1}(b)=\gamma(b)$ satisfies:

$$
\begin{aligned}
u(\gamma(b))-u(\gamma(a)) & =\int_{a}^{b} d_{\gamma_{1}(s)} u\left(\gamma_{1}(s)\right) \dot{\gamma}(s) d s \\
& \leq \int_{a}^{b}\left[L_{\eta}\left(\gamma_{1}(s), \dot{\gamma}_{1}(s)\right)+H_{\eta}\left(\gamma_{1}(s), d_{\gamma_{1}(s)} u\right)\right] d s
\end{aligned}
$$

where the second inequality follows again by the duality between Hamiltonian and Lagrangian. Note that now $H_{\eta}\left(\gamma_{1}(s), d_{\gamma_{1}(s)} u\right) \leq \alpha(c)$, because $\Lambda=\{(x, \eta(x)+$ $d u(x))\}$ is subcritical. Then

$$
\int_{a}^{b} L_{\eta}\left(\gamma_{1}(s), \dot{\gamma}_{1}(s)\right) d s \geq u(\gamma(b))-u(\gamma(a))-\alpha(c)(b-a)
$$

and this proves that $\gamma$ is a $c$-minimizer.

Let us finally observe that the Hamilton function on any invariant Lagrangian graph $\Lambda=\{(x, \eta+d u)\}$ is a constant: $H(x, \eta+d u)=k$ (the classical proof easily extends to the case of Lipschitz Lagrangian graphs, see for instance [26]). Then $u$ is a classical solution of the Hamilton-Jacobi equation (corresponding to the cohomology class $c$ ). As showed in $[10,18]$, there is only one possible value of $k$ for which such solutions can exist, namely $k=\alpha(c)$, and this shows that $\Lambda$ coincides with its critical part. By the result proved in item (ii), if $\mu^{*}$ is supported on $\Lambda$, then $\mu$ is a $c$-action minimizing measure and this proves the last claim in the statement of the lemma.

We can now prove the well-known uniqueness result for Lagrangian graphs supporting invariant measures of full support, in a fixed cohomology class, stated in Section 2.

Theorem 3.2. If $\Lambda \subset \mathrm{T}^{*} M$ is a Lagrangian graph on which the Hamiltonian $d y$ namics admits an invariant measure $\mu^{*}$ with full support, then $\Lambda=\mathcal{L}\left(\widetilde{\mathcal{M}}_{c}\right)=$ $\mathcal{L}\left(\tilde{\mathcal{A}}_{c}\right)$, where $c$ is the cohomology class of $\Lambda$. Therefore, if $\Lambda_{1}$ and $\Lambda_{2}$ are two Lagrangian graphs as above, with the same cohomology class, then $\Lambda_{1}=\Lambda_{2}$.

This theorem can be also obtained as a corollary of the results in [20, Appendix 2]. Our proof is essentially a rehash of the same ideas, using a different point of view. In fact the Weierstrass method, used in [20] to show that orbits on a KAM torus are action minimizing, or the use of Hamilton-Jacobi equation are essentially two sides of the same coin.

Proof. By Lemma 3.1, the measure $\mu=\mathcal{L}_{*}^{-1} \mu^{*}$ is $c$-action minimizing. This means that $\mathcal{L}^{-1}(\Lambda)=\operatorname{supp} \mu \subseteq \widetilde{\mathcal{M}}_{c} \subseteq \widetilde{\mathcal{A}}_{c}$. Note however that $\widetilde{\mathcal{M}}_{c}$ and $\widetilde{\mathcal{A}}_{c}$ are graphs and, since supp $\mu$ is a graph over the whole $M$, it follows that

$$
\mathcal{L}^{-1}(\Lambda)=\operatorname{supp} \mu=\widetilde{\mathcal{M}}_{c}=\widetilde{\mathcal{A}}_{c} .
$$


One can deduce something more from the above proof. Recall the definition of Mather's $\beta$-function: $\beta(h)$ is the minimum of the average action $\int L d \mu$ over the invariant measures $\mu$ with a given rotation vector $h$ [20]. This function is convex and its conjugate function (given by Fenchel's duality) coincides with the $\alpha$-function: $\alpha(c)=\max _{h \in \mathrm{H}_{1}(M ; \mathbb{R})}(\langle c, h\rangle-\beta(h))$.

Theorem 3.3. If $\Lambda$ and $\mu$ are as in Theorem 3.2 and $\rho$ is the rotation vector of $\mu=\mathcal{L}^{-1} \mu^{*}$, then $\Lambda=\mathcal{L}\left(\widetilde{\mathcal{M}}^{\rho}\right)$. Therefore, if $\Lambda_{1}$ and $\Lambda_{2}$ are two Lagrangian graphs supporting measures of full support and the same rotation vector $\rho$, then $\Lambda_{1}=\Lambda_{2}$. Moreover, Mather's $\beta$-function is differentiable at $\rho$ with $\partial \beta(\rho)=c$, where $c$ is the cohomology class of $\Lambda$.

Proof. The first claim follows from the fact that $\widetilde{\mathcal{M}}^{\rho}$ is a graph over $M$ and that by definition $\widetilde{\mathcal{M}}^{\rho} \supseteq \operatorname{supp} \mu=\mathcal{L}^{-1}(\Lambda)$. As far as the differentiability of $\beta$ at $\rho$ is concerned, suppose that $c^{\prime} \in \mathrm{H}^{1}(M ; \mathbb{R})$ is a subderivative of $\beta$ at $\rho$. Then, using Fenchel's duality and the fact that $\alpha$ and $\beta$ are conjugated, $\beta(\rho)=\left\langle c^{\prime}, \rho\right\rangle-\alpha\left(c^{\prime}\right)$ and this implies that each Mather's measure $\mu$ with rotation vector $\rho$ is also $c^{\prime}-$ action minimizing; in fact:

$$
\begin{aligned}
\int_{\mathrm{T} M}\left(L(x, v)-\left\langle\eta^{\prime}(x), v\right\rangle\right) d \mu & =\int_{\mathrm{T} M} L(x, v) d \mu-\int_{\mathrm{T} M}\left\langle\eta^{\prime}(x), v\right\rangle d \mu \\
& =\beta(\rho)-\left\langle c^{\prime}, \rho\right\rangle=-\alpha\left(c^{\prime}\right),
\end{aligned}
$$

where $\eta^{\prime}$ is a closed 1 -form of cohomology $c^{\prime}$. As a result, $\widetilde{\mathcal{M}}^{\rho}=\mathcal{L}^{-1}(\Lambda) \subseteq \widetilde{\mathcal{M}}_{c^{\prime}}$. The graph property of $\widetilde{\mathcal{M}}_{c^{\prime}}$ and of $\widetilde{\mathcal{A}}_{c^{\prime}}$ implies that $\widetilde{\mathcal{A}}_{c^{\prime}}=\widetilde{\mathcal{M}}_{c^{\prime}}=\mathcal{L}^{-1}(\Lambda)$ and therefore $\mathcal{L}\left(\widetilde{\mathcal{A}}_{c^{\prime}}\right)=\Lambda$. Since the cohomology class of $\Lambda$ is $c$, it follows that $c^{\prime}=c$.

We are now in the position of proving the main uniqueness result in a homology class, stated in Section 2.

Theorem 3.4. Let $\Lambda$ be a Schwartzman strictly ergodic invariant Lagrangian graph with homology class $\rho$. The following properties are satisfied:

(i) if $\Lambda \cap \mathcal{L}\left(\tilde{\mathcal{A}}_{c}\right) \neq \emptyset$, then $\Lambda=\mathcal{L}\left(\tilde{\mathcal{A}}_{c}\right)$ and $c=c_{\Lambda}$, where $c_{\Lambda}$ is the cohomology class of $\Lambda$.

(ii) the Mather function $\alpha$ is differentiable at $c_{\Lambda}$ and $\partial \alpha\left(c_{\Lambda}\right)=\rho$.

Therefore,

(iii) any invariant Lagrangian graph that carries a measure with rotation vector $\rho$ is equal to the graph $\Lambda$;

(iv) any invariant Lagrangian graph is either disjoint from $\Lambda$ or equal to $\Lambda$.

We shall need the following lemma. 
Lemma 3.5. Let $\rho$, c be respectively an arbitrary homology class in $\mathrm{H}_{1}(M ; \mathbb{R})$ and an arbitrary cohomology class in $\mathrm{H}^{1}(M ; \mathbb{R})$. We have
(1) $\widetilde{\mathcal{M}}^{\rho} \cap \widetilde{\mathcal{M}}_{c} \neq \emptyset$
(2) $\widetilde{\mathcal{M}}^{\rho} \subseteq \widetilde{\mathcal{M}}_{c}$
(3) $\rho \in \partial \alpha(c)$.

Proof of Lemma 3.5. The implication (2) $\Longrightarrow(1)$ is trivial. Let us prove that $(1) \Longrightarrow(3)$. If $\widetilde{\mathcal{M}}^{\rho} \cap \widetilde{\mathcal{M}}_{c} \neq 0$, then there exists a $c$-action minimizing invariant measure $\mu$ with rotation vector $\rho$. Let $\eta$ be a closed 1 -form with $[\eta]=c$; from the definition of $\alpha$ and $\beta$ :

$$
\begin{aligned}
-\alpha(c) & =\int_{\mathrm{T} M}(L(x, v)-\langle\eta(x), v\rangle) d \mu \\
& =\int_{\mathrm{T} M} L(x, v) d \mu-\langle c, \rho\rangle=\beta(\rho)-\langle c, \rho\rangle ;
\end{aligned}
$$

since $\beta$ and $\alpha$ are convex conjugated, then $\rho$ is a subderivative of $\alpha$ at $c$.

Finally, in order to show (3) $\Longrightarrow(2)$, let us prove that any Mather's measure with rotation vector $\rho$ is $c$-action minimizing. In fact, if $\rho \in \partial \alpha(c)$ then $\alpha(c)=$ $\langle c, \rho\rangle-\beta(\rho)$; therefore for any $\mu$ is a Mather's measure with rotation vector $\rho$ and $\eta$ as above:

$$
-\alpha(c)=\beta(\rho)-\langle c, \rho\rangle=\int_{\mathrm{T} M}(L(x, v)-\langle\eta(x), v\rangle) d \mu .
$$

This proves that $\mu$ is $c$-action minimizing and concludes the proof.

Proof of Theorem 3.4. (i) From Theorem 3.2, it follows that $\mathcal{L}^{-1}(\Lambda)=\widetilde{\mathcal{A}}_{c_{\Lambda}}$. Let us show that it does not intersect any other Aubry set. Suppose by contradiction that $\mathcal{L}^{-1}(\Lambda)$ intersects another Aubry set $\widetilde{\mathcal{A}}_{c}$. By Theorem $3.3, \mathcal{L}^{-1}(\Lambda)=\widetilde{\mathcal{M}}^{\rho}$, then $\widetilde{\mathcal{M}}^{\rho} \cap \widetilde{\mathcal{A}}_{c} \neq \emptyset$ and, because of Lemma 3.1, Lemma 3.5 and the graph property of $\widetilde{\mathcal{A}}_{c}$, we can conclude that $\widetilde{\mathcal{A}}_{c}=\mathcal{L}^{-1}(\Lambda)$. The same argument used in the proof of Theorem 3.3 allows us to conclude that $c=c_{\Lambda}$.

(ii) Suppose that $h \in \partial \alpha\left(c_{\Lambda}\right)$. The previous lemma implies that $\widetilde{\mathcal{M}}^{h} \subseteq \Lambda$; the Schwartzman unique ergodicity property of $\Lambda$ implies $h=\rho$. Therefore $\alpha$ is differentiable at $c_{\Lambda}$ and $\partial \alpha\left(c_{\Lambda}\right)=\rho$.

To prove (iv), let $\Lambda_{1}$ be an invariant Lagrangian graph, and call $c_{1}$ its cohomology class. If the compact invariant set $\Lambda \cap \Lambda_{1}$ is not empty, then we can find a probability measure $\mu^{*}$ invariant under the flow and whose support is contained in this intersection. Since $\mu^{*}$ is contained in the Lagrangian graph $\Lambda_{1}$, by Lemma 3.1, it is $c_{1}$-action minimizing. Hence, the support of $\mu^{*}$ is contained in $\mathcal{L}\left(\widetilde{\mathcal{A}}_{c_{1}}\right)$. This shows that the intersection $\Lambda \cap \mathcal{L}\left(\widetilde{\mathcal{A}}_{c_{1}}\right)$ contains the support of $\mu^{*}$ and is therefore not empty. By $(i), \Lambda=\mathcal{L}\left(\widetilde{\mathcal{A}}_{c_{1}}\right)$. Moreover, note that $\mathcal{L}\left(\widetilde{\mathcal{A}}_{c_{1}}\right) \subseteq \Lambda_{1}$, because $\Lambda_{1}=\operatorname{Graph}\left(\eta_{1}+d u_{1}\right)$, with $\left[\eta_{1}\right]=c_{1}$ and $u_{1}$ a classical solution to the HamiltonJacobi equation (see [10]). Therefore, $\Lambda=\Lambda_{1}$, since they are both graphs over $M$. 
To prove (iii), consider an invariant Lagrangian graph $\Lambda_{1}$, with cohomology class $c_{1}$, which carries an invariant measure $\mu^{*}$ whose rotation vector is $\rho$. By Lemma 3.1, the measure $\mu^{*}$ is $c_{1}$-minimizing. Therefore, we have $\widetilde{\mathcal{M}}^{\rho} \cap \widetilde{\mathcal{M}}_{c_{1}} \neq \emptyset$. By Lemma 3.5, it follows that $\mathcal{L}^{-1}(\Lambda)=\widetilde{\mathcal{M}^{\rho}} \subseteq \widetilde{\mathcal{M}}_{c_{1}} \subseteq \widetilde{\mathcal{A}}_{c_{1}} \subseteq \mathcal{L}^{-1}\left(\Lambda_{1}\right)$. Again, this forces the equality $\Lambda=\Lambda_{1}$ by the graph property.

Finally, observe that using Lemma 3.5, one can also deduce the following property.

Corollary 3.6. Mather's $\alpha$-function is differentiable at $c$ if and only if the restriction of the Euler-Lagrange flow to $\widetilde{\mathcal{A}}_{c}$ is Schwartzman uniquely ergodic, i.e. if and only if all invariant measures supported on $\widetilde{\mathcal{A}}_{c}$ have the same rotation vector.

\section{Global uniqueness of KAM tori}

In this section we motivate more precisely the problem of uniqueness of KAM tori and prove Corollary 2.6. We also show how to generalize Corollary 2.6 to cover the case of invariant tori belonging to the closure of the set of KAM tori.

KAM theory concerns the study of existence of KAM tori (see Definition 2.5) in quasi-integrable Hamiltonian systems of the form $H(x, p)=H_{0}(p)+\varepsilon f(x, p)$, where: $(x, p)$ are local coordinates on $\mathbb{T}^{n} \times \mathbb{R}^{n}, \varepsilon$ is a "small" parameter and $f(x, p)$ a smooth function. If $\varepsilon=0$ the system is integrable, in the sense that the dynamics can be explictly solved: in particular each torus $\mathbb{T}^{n} \times\left\{p_{0}\right\}$ is invariant and the motion on it corresponds to a rotation with frequency $\rho\left(p_{0}\right)=\frac{\partial H_{0}}{\partial p}\left(p_{0}\right)$. The question addressed by KAM theory is whether this foliation of phase space into invariant tori, on which the motion is (quasi-)periodic, persists even if $\varepsilon \neq 0$. In 1954 Kolmogorov stated (and Arnol'd [1] and Moser [22] proved it later in different contexts) that, in spite of the generic disappearence of the invariant submanifolds filled by periodic orbits, pointed out by Poincaré, for small $\varepsilon$ it is always possible to find KAM tori corresponding to "strongly non-resonant", i.e. Diophantine, rotation vectors. The celebrated KAM Theorem (in one of its several versions) not only shows the existence of such tori, but also provides an explicit method to construct them.

Theorem 4.1 (Kolmogorov-Arnol'd-Moser [24]). Let $n \geq 2, \tau>n-1, C>0$, $\ell>2 \tau+2, M>0$ and $r>0$ be given. Let $B_{r} \in \mathbb{R}^{n}$ be the open ball of radius $r$ centered at the origin. Let $H \in C^{\ell}\left(\mathbb{T}^{n} \times B_{r}\right)$ be of the form

$$
H(x, p)=H_{0}(p)+\varepsilon f(x, p)
$$

with $\left|H_{0}\right|_{C^{\ell}} \leq M,|f|_{C^{\ell}} \leq M,\left|\frac{\partial^{2} H_{0}}{\partial p^{2}}\right| \geq M^{-1}$ and $\rho=\frac{\partial H_{0}}{\partial p}(0) \in \mathcal{D}(C, \tau)$. Then, for any $s<\ell-2 \tau-1$, there exists $\varepsilon_{0}>0$ such that for any $\varepsilon \leq \varepsilon_{0}$ the Hamiltonian (4.1) admits a $C^{s, s+\tau}$ KAM torus with rotation vector $\rho$, i.e. a $C^{s+\tau}$ invariant torus such that the Hamiltonian flow on it is $C^{s}$-conjugated with a rotation with frequency $\rho$. 
The invariant torus constructed in the proof of the KAM Theorem is locally unique, in the sense that for any prescribed (and admissible) $s$ there is at most one $C^{s, s+\tau}$ KAM torus with rotation vector $\rho$ within a $C^{s}$-distance $\delta(n, s, C, \tau)$ to the one constructed in the proof of the KAM Theorem, see [5, 23, 24]. Note that the $C^{s}$-distance $\delta$ within which one can prove uniqueness of the KAM torus in a prescribed regularity class depends both on the irrationality properties of $\rho$ and on the regularity class $s$ itself. It is then a priori possible that even for small $\varepsilon$ there exist different KAM tori within a prescribed $C^{1}$-distance from the one constructed in the proof of the Theorem, possibly less regular than that torus. Quite surprisingly, even in the analytic case, we are not aware of any proof of "global" uniqueness of the invariant analytic KAM torus with rotation vector $\rho$ (of course in the analytic case the analytic torus one manages to construct is unique within the class of analytic tori - however nothing a priori guarantees that less regular invariant tori with the same rotation vector exist).

Our result, in the form stated in Corollary 2.6, settles the question and shows that, at least in the case of Tonelli Hamiltonians, it is not possible to have two different KAM tori with the same rotation vector. Note that the assumption of strict convexity of the Hamiltonian is necessary to exclude trivial sources of nonuniqueness: for instance, in the context of quasi-integrable Hamiltonians, global uniqueness could be lost simply because the unperturbed Hamiltonian induces a map $p \mapsto \partial_{p} H_{0}(p)$ from actions to frequencies that is not one to one. Let us also remark that, apparently, the Hamiltonian considered in KAM Theorem is not a Tonelli Hamiltonian, since the latter, by definition, is defined globally on the whole $\mathbb{T}^{n} \times \mathbb{R}^{n}$. However any $C^{\ell}$ strictly convex Hamiltonian defined on $\mathbb{T}^{n} \times B_{r}$ for some $r>0$ can be extended to a global $C^{\ell}$ Tonelli Hamiltonian. Then in the statement of the KAM Theorem above it is actually enough to assume $H$ to be a $C^{\ell}$ Tonelli Hamiltonian, locally satisfying the (in)equalities listed after (4.1).

Given the proof of our main results in Section 3, the proof of Corollary 2.6 becomes very simple.

Proof of Corollary 2.6. Since the Lagrangian KAM torus $\mathcal{T}$ admits an invariant measure $\mu^{*}$ of full support, which is the image via the conjugation $\varphi$ of the uniform measure on $\mathbb{T}^{n}$, then the claims follow from Theorem 3.3. Note that for rationally independent rotation vectors, a classical remark by Herman [15] implies that $\mathcal{T}$ is automatically Lagrangian (it is sufficient that the flow on it is topologically conjugated to a transitive flow on $\mathbb{T}^{n}$ ).

An interesting generalization of the result of Corollary 2.6 concerns the invariant tori belonging to the $C^{0}$-closure $\bar{\Upsilon}$ of the set $\Upsilon$ of all Lagrangian KAM tori. Note that, for quasi-integrable systems, $\Upsilon$ is not empty. The set $\Upsilon$ can be seen as a subset of $\operatorname{Lip}\left(\mathbb{T}^{n}, \mathbb{R}^{n}\right)$. This follows from Theorem 3.3, and from Mather's graph theorem and the other results in [20]. Moreover, any family of invariant Lagrangians graphs on which the function $\alpha$ (or $H$ ) is bounded gives rise to a family of functions in $\operatorname{Lip}\left(\mathbb{T}^{n}, \mathbb{R}^{n}\right)$ with uniformly bounded Lipschitz constant. This is because, given $\Lambda$ in such a family and denoting by $(\eta+d u)$ its graph, for any pair 
of points $x, y \in \mathbb{T}^{n}$ and any smooth curve $\gamma(t)$ on $\Lambda$ connecting $x$ to $y$ with unit speed, we have that $u(x)-u(y) \leq \int_{0}^{|x-y|} L_{\eta}(\gamma(t), \dot{\gamma}(t))+\alpha(c)|x-y|$, where $c=[\eta]$, see (3.4). By the Ascoli-Arzelà theorem, it follows that $\bar{\Upsilon}$ is also a subset of $\operatorname{Lip}\left(\mathbb{T}^{n}, \mathbb{R}^{n}\right)$, consisting of functions whose graphs are invariant (Lipschitz) Lagrangian tori. Herman [16] showed that, for a generic Hamiltonian $H$ close enough to an integrable Hamiltonian $H_{0}$, the dynamics on the generic tori in $\bar{\Upsilon}$ is not conjugated to a rotation. These "new" tori therefore represent the majority, in the sense of topology, and hence most invariant tori cannot be obtained by the KAM algorithm. More precisely, Herman showed that in $\bar{\Upsilon}$ there exists a dense $G_{\delta}$-set (i.e. a dense countable intersection of open sets) of invariant Lagrangian graphs on which the dynamics is strictly ergodic and weakly mixing, and for which the rotation vector, in the sense of Section 2, is not Diophantine. These invariant graphs are therefore not obtained by the KAM theorem, however our uniqueness result do still apply to these graphs since strict ergodicity implies Schwartzman strict ergodicity.

More generally, given any Tonelli Lagrangian on $\mathbb{T}^{n}$, we consider the set $\tilde{\Upsilon}$ of invariant Lagrangian graphs on which the dynamics of the flow is topologically conjugated to an ergodic linear flow on $\mathbb{T}^{n}$ (of course, far from the canonical integrable Lagrangian the set $\tilde{\Upsilon}$ may be empty). The dynamics on anyone of the invariant graphs in $\tilde{\Upsilon}$ is strictly ergodic. Since the set of strictly ergodic flows on a compact set is a $G_{\delta}$-set in the $C^{0}$ topology, see for example [11, Corollaire 4.5], it follows that there exists a dense $G_{\delta}$-subset $\mathcal{G}$ of the $C^{0}$ closure of $\tilde{\Upsilon}$ in $\operatorname{Lip}\left(\mathbb{T}^{n}, \mathbb{R}^{n}\right)$, such that the dynamics on any $\Lambda \in \mathcal{G}$ is strictly ergodic. Therefore we get the following proposition.

Proposition 4.2. There exists a dense $G_{\delta}$-set $\mathcal{G}$ in the $C^{0}$ closure of $\tilde{\Upsilon}$ consisting of strictly ergodic invariant Lagrangian graphs. Any $\Lambda \in \mathcal{G}$ satisfies the following properties:

(i) the invariant graph $\Lambda$ has a well-defined rotation vector $\rho(\Lambda)$.

(ii) Any invariant Lagrangian graph that intersects $\Lambda$ coincides with $\Lambda$.

(iii) Any Lagrangian invariant graph that carries an invariant measure whose rotation is $\rho(\Lambda)$ coincides with $\Lambda$.

\section{Appendix A. Schwartzman unique and strict ergodicity}

In this section we prove some results on the structure of the set of Schwartzman uniquely/strictly ergodic graphs that we have introduced in Section 2, and provide some examples.

Let us start from the notion of Schwartzman asymptotic cycle of a flow, introduced by Sol Schwartzman in [25], as a first attempt to develop an algebraic topological approach to the study of dynamics. This is closely related to the concept of rotation vector of a measure, that we have introduced in Section 2. We shall give a different description of the Schwartzman asymptotic cycle of a flow using the flux homomorphism in volume preserving and symplectic geometry (see [2, Chapter 3]), from the same perspective as [9]. The definition used below has the technical 
advantage of not relying on the Krylov-Bogolioubov theory of generic orbits in a dynamical system, although a more geometrical definition showing that "averaged" pieces of long orbits converge almost everywhere in the first homology group for any invariant measure is certainly more heuristic and intuitive.

Let $X$ be a topological space and $\left(\phi_{t}\right)_{t \in \mathbb{R}}$ a continuous flow on $X$. We shall define $\Phi: X \times[0,1] \rightarrow X$ by $\Phi(x, t)=\phi_{t}(x)$. Consider a continuous function $f: X \rightarrow \mathbb{T}$ and let $F(f, \Phi): X \times[0,1] \rightarrow \mathbb{T}$ be

$$
F(f, \Phi)(x, t)=f\left(\phi_{t}(x)\right)-f(x) .
$$

$F(f, \Phi)$ is continuous and identically 0 on $X \times\{0\}$, it is therefore homotopic to a constant and can be lifted to a continuous map $\bar{F}(f, \Phi): X \times[0,1] \rightarrow \mathbb{R}$, with $\bar{F}(f, \Phi) \mid X \times\{0\}$ identically 0 . We define

$$
\mathcal{V}\left(f, \phi_{t}\right)(x):=\bar{F}(f, \Phi)(x, 1) .
$$

Note that if $f$ is homotopic to 0 then it can be lifted continuously to $\bar{f}: X \rightarrow \mathbb{R}$. In that case $\bar{F}(f, \Phi)=\bar{f} \Phi-\bar{f}$, and

$$
\mathcal{V}\left(f, \phi_{t}\right)(x)=\bar{f}\left(\phi_{1}(x)\right)-\bar{f} .
$$

If $\mu$ is a measure with compact support invariant under the flow $\phi_{t}$, for a continuous $f: X \rightarrow \mathbb{T}$, we define $\mathcal{S}\left(\mu, \phi_{t}\right)(f)$, or simply $\mathcal{S}(\mu)(f)$ when $\phi_{t}$ is fixed, by

$$
\mathcal{S}(\mu)(f)=\int_{X} \mathcal{V}\left(f, \phi_{t}\right)(x) d \mu(x) .
$$

If we denote by $[X, \mathbb{T}]$ the set of homotopy classes of continuous maps from $X$ to $\mathbb{T}$, it is not difficult to verify that $\mathcal{S}(\mu)$ is a well-defined additive homomorphism from the additive group $[X, \mathbb{T}]$ to $\mathbb{R}$.

When $X$ is a good space (like a manifold or a locally finite polyhedron), it is well-known that $[X, \mathbb{T}]$ is canonically identified with the first cohomology group $\mathrm{H}^{1}(X ; \mathbb{Z})$. In that case $\mathcal{S}(\mu)$ is in $\operatorname{Hom}\left(\mathrm{H}^{1}(X ; \mathbb{Z}), \mathbb{R}\right)$. Since the first cohomology group with real coefficients $\mathrm{H}^{1}(X ; \mathbb{R})$ is $\mathrm{H}^{1}(X ; \mathbb{Z}) \otimes \mathbb{R}$, we can view $\mathcal{S}(\mu)$ as an element of the dual $\mathrm{H}^{1}(X ; \mathbb{R})^{*}$ of the $\mathbb{R}$-vector space $\mathrm{H}^{1}(X ; \mathbb{R})$. When $\mathrm{H}^{1}(X ; \mathbb{R})$ is finite-dimensional (for instance, when $X$ is a finite polyhedron or a compact manifold) then $\mathrm{H}^{1}(X ; \mathbb{R})^{*}$ is in fact equal to the first homology group $\mathrm{H}_{1}(X ; \mathbb{R})$, and therefore $\mathcal{S}(\mu)$ defines an element of $\mathrm{H}_{1}(X ; \mathbb{R})$, i.e. a 1-cycle. This 1-cycle $\mathcal{S}(\mu)$ is called the Schwartzman asymptotic cycle of $\mu$.

Let us now consider the case of a $C^{1}$ flow $\phi_{t}$ on a manifold $N$. We call $X$ the continuous vector field on $N$ generating $\phi_{t}$, i.e.

$$
\forall x \in N, \quad X(x)=\left.\frac{d \phi_{t}(x)}{d t}\right|_{t=0} .
$$

By the flow property $\phi_{t+t^{\prime}}=\phi_{t} \circ \phi_{t^{\prime}}$, this implies

$$
\forall x \in N, \forall t \in \mathbb{R}, \quad \frac{d \phi_{t}(x)}{d t}=X\left(\phi_{t}(x)\right) .
$$


In the case of a manifold $N$, the identification of $[N, \mathbb{T}]$ with $\mathrm{H}^{1}(N ; \mathbb{Z})$ is best described with the de Rham cohomology. We consider the natural map $I_{N}:[N, \mathbb{T}] \rightarrow$ $\mathrm{H}^{1}(N ; \mathbb{R})$ defined by

$$
I_{N}([f])=\left[f^{*} \theta\right],
$$

where $[f]$ on the left-hand side denotes the homotopy class of the $C^{\infty}$ map $f$ : $N \rightarrow \mathbb{T}$, and $\left[f^{*} \theta\right]$ on the right hand side is the cohomology class of the pullback by $f$ of the closed 1 -form on $\mathbb{T}$ whose lift to $\mathbb{R}$ is $d t$. Note that any homotopy class in $[N, \mathbb{T}]$ contains smooth maps because $C^{\infty}$ maps are dense in $C^{0}$ maps (for the Whitney topology). Therefore the map $I_{N}$ is indeed defined on the whole of $[N, \mathbb{T}]$. As it is well-known, this map $I_{N}$ induces an isomorphism of $[N, \mathbb{T}]$ on $\mathrm{H}^{1}(N ; \mathbb{Z}) \subset \mathrm{H}^{1}(N ; \mathbb{R})=\mathrm{H}^{1}(N ; \mathbb{Z}) \otimes \mathbb{R}$.

Given a $C^{\infty}$ map $f: N \rightarrow \mathbb{T}$, the $C^{1}$ flow $\phi_{t}$ on $N$, and $x \in N$, we compute $\mathcal{V}\left(f, \phi_{t}\right)(x)$. If $\gamma_{x}:[0,1] \rightarrow N$ is the path $t \mapsto \phi_{t}(x)$, since $\gamma_{x}$ is $C^{1}$, we get

$$
\mathcal{V}\left(f, \phi_{t}\right)(x)=\int_{f \circ \gamma_{x}} \theta=\int_{\gamma_{x}} f^{*} \theta .
$$

Moreover, since $\gamma_{x}(t)=\phi_{t}(x)$, we have $\dot{\gamma}_{x}(t)=X\left(\phi_{t}(x)\right)$. It follows that

$$
\mathcal{V}\left(f, \phi_{t}\right)(x)=\int_{0}^{1}\left(f^{*} \theta\right)_{\phi_{t}(x)}\left(X\left[\phi_{t}(x)\right]\right) d t=\int_{0}^{1}\left(i_{X} f^{*} \theta\right)\left(\phi_{t}(x)\right) d t,
$$

where $i_{X}$ denotes the interior product of a differential form with $X$. Therefore if $\mu$ is an invariant measure for $\phi_{t}$, which we shall assume to have a compact support, we obtain

$$
\begin{aligned}
\mathcal{S}(\mu) & =\int_{N} \int_{0}^{1}\left(i_{X} f^{*} \theta\right)\left(\phi_{t}(x)\right) d t d \mu(x)=\int_{0}^{1} \int_{N}\left(i_{X} f^{*} \theta\right)\left(\phi_{t}(x)\right) d \mu(x) d t \\
& =\int_{0}^{1} \int_{N}\left(i_{X} f^{*} \theta\right)(x) d \mu(x) d t=\int_{N}\left(i_{X} f^{*} \theta\right) d \mu .
\end{aligned}
$$

This shows that as an element of $\mathrm{H}^{1}(M ; \mathbb{R})^{*}$, the Schwarztman asymptotic cycle $\mathcal{S}(\mu)$ is given by

$$
\mathcal{S}(\mu)([\eta])=\int_{N} i_{X} \eta d \mu .
$$

We would like now to relate the Schwartzman asymptotic cycles to the rotation vectors $\rho(\mu)$ defined in section 2 for Euler-Lagrange flows. In this case $N=T M$ and $\phi_{t}$ is an Euler-Lagrange flow $\phi_{t}^{L}$ of some Lagrangian $L$. If we call $X_{L}$ the vector field generating $\phi_{t}^{L}$, since this flow is obtained from a second order ODE on $M$, we get

$$
\forall x \in M, \forall v \in T_{x} M, \quad T \pi\left(X_{L}(x, v)\right)=v,
$$

where $T \pi: T(T M) \rightarrow T M$ denotes the canonical projection. Since this projection $\pi$ is a homotopy equivalence, to compute $\mathcal{S}(\mu)$ we only need to consider 
forms of the type $\pi^{*} \eta$ where $\eta$ is a closed 1 -form on the base $M$. In this case $\left(i_{X_{L}} \pi^{*} \eta\right)(x, v)=\eta_{x}\left(T \pi\left(X_{L}(x, v)\right)=\eta_{x}(v)\right.$. Therefore, for any probability measure $\mu$ on $T M$ with compact support and invariant under $\phi_{t}^{L}$, we obtain

$$
\mathcal{S}(\mu)\left[\pi^{*} \eta\right]=\int_{T M} \eta_{x}(v) d \mu(x, v)=\int_{T M}\langle\eta, v\rangle d \mu .
$$

This is precisely $\rho(\mu)$ as it was defined above in section 2 . Note that the only property we have used is the fact that $\phi_{t}$ is the flow of a second order ODE on the base $M$.

\section{Examples}

- We can now easily compute Schwartzman asymptotic cycles for linear flows on $\mathbb{T}^{n}$. Such a flow is determined by a constant vector field $\alpha \in \mathbb{R}^{n}$ on $\mathbb{T}^{n}$ (here we use the canonical trivialization of the tangent bundle of $\mathbb{T}^{n}$ ), the associated flow $R_{t}^{\alpha}: \mathbb{T}^{n} \rightarrow \mathbb{T}^{n}$ is defined by $R_{t}^{\alpha}(x)=x+[t \alpha]$, where $[t \alpha]$ is the class in $\mathbb{T}^{n}=\mathbb{R}^{n} / \mathbb{Z}^{n}$ of the vector $t \alpha \in \mathbb{R}^{n}$. If $\omega$ is a 1 -form with constant coefficients, i.e. $\omega=\sum_{i=1}^{n} a_{i} d x_{i}$, with $a_{i} \in \mathbb{R}$, the interior product $i_{\alpha} \omega$ is the constant function $\sum_{i=1}^{n} \alpha_{i} a_{i}$. Therefore, it follows that $\mathcal{S}(\mu)=\alpha \in \mathbb{R}^{n} \equiv \mathrm{H}_{1}\left(\mathbb{T}^{n} ; \mathbb{R}\right)$.

- Suppose that $x$ is a periodic point of $\phi_{t}$ or period $T>0$. One can define an invariant probability measure $\mu_{x, t_{0}}$ for $\phi_{t}$ by

$$
\int_{X} g(x) d \mu_{x, t_{0}}=\frac{1}{t_{0}} \int_{0}^{t_{0}} g\left(\left(\phi_{t}(x)\right) d t,\right.
$$

where $g: X \rightarrow \mathbb{R}$ is a measurable function. We let the reader verify that $\mathcal{S}\left(\mu_{x, t_{0}}\right)$ is equal in $\mathrm{H}_{1}(X ; \mathbb{R})$ to the homology class $\left[\gamma_{x, t_{0}}\right] / t_{0}$, where $\gamma_{x, t_{0}}$ is the loop $t \mapsto \phi_{t}(x), t \in\left[0, t_{0}\right]$.

- When $x$ is a fixed point of $\phi_{t}$, then the Dirac mass $\delta_{x}$ at $x$ is invariant under $\phi_{t}$, and in that case $\mathcal{S}\left(\delta_{x}\right)=0$.

Let us now study the behavior of Schwartzman asymptotic cycles under semiconjugacy.

Proposition A.1. Suppose $\phi_{t}^{i}: X_{i} \rightarrow X_{i}, i=1,2$ are two continuous flows. Suppose also that $\psi: X_{1} \rightarrow X_{2}$ is a continuous semi-conjugation between the flows, i.e. $\psi \circ \phi_{t}^{1}=\phi_{t}^{2} \circ \psi$, for every $t \in \mathbb{R}$. Given a probability measure $\mu$ with compact support on $X_{1}$ invariant under $\phi_{t}^{1}$, then, for every continuous map $f: X_{2} \rightarrow \mathbb{T}$, we have

$$
\mathcal{S}\left(\psi_{*} \mu, \phi_{t}^{2}\right)([f])=\mathcal{S}\left(\mu, \phi_{t}^{1}\right)([f \circ \psi]),
$$

where $\psi_{*} \mu$ is the image of $\mu$ under $\psi$. In particular, if we are in the situation where $\operatorname{Hom}\left(\left[X_{i}, \mathbb{T}\right]\right) \equiv \mathrm{H}_{1}\left(X_{i} ; \mathbb{R}\right), i=1,2$, we obtain

$$
\mathcal{S}\left(\psi_{*} \mu, \phi_{t}^{2}\right)=H_{1}(\psi)\left(\mathcal{S}\left(\mu, \phi_{t}^{1}\right)\right) .
$$


Proof. Notice that $f \psi \phi_{t}^{1}(x)-f \psi(x)=f \phi_{t}^{2}(\psi(x))-f(\psi(x))$. Therefore by uniqueness of liftings $\mathcal{V}\left(f \psi, \phi_{t}^{1}\right)(x)=\mathcal{V}\left(f, \phi_{t}^{2}\right)(\psi(x))$. An integration with respect to $\mu$ finishes the proof.

To simplify things, in the remainder of this appendix, we shall assume that $X$ is a compact space, for which we have $[X, \mathbb{T}]=\mathrm{H}^{1}(X ; \mathbb{Z})$, and $\mathrm{H}_{1}(X ; \mathbb{Z})$ is finitely generated. In that case, the dual space $\mathrm{H}^{1}(X ; \mathbb{R})^{*}$ is $\mathrm{H}_{1}(X ; \mathbb{R})$, and for every flow $\phi_{t}$ on $X$ and every probability measure $\mu$ on $X$ invariant under $\phi_{t}$, the Schwartzman asymptotic cycle is an element of the finite dimensional-vector space $\mathrm{H}_{1}(X ; \mathbb{R})$.

Definition A.2. For a flow $\phi_{t}$ on $X$, we denote by $\mathcal{S}\left(\phi_{t}\right)$ the set of all Schwartzman asymptotic cycles $\mathcal{S}(\mu)$, where $\mu$ is an arbitrary probability measure on $X$ invariant under $\phi_{t}$.

Since $X$ is compact, note that for the weak topology the set $\mathfrak{M}(X)$ of probability Borel measures on $X$ is compact and convex. It is even metrizable, since we are assuming $X$ metrizable. Furthermore the subset $\mathfrak{M}\left(X, \phi_{t}\right) \subseteq \mathfrak{M}(X)$ of probability measures invariant under $\phi_{t}$ is, as it is well-known, compact convex and non empty. Therefore $\mathcal{S}\left(\phi_{t}\right)$ is a compact convex non-empty subset of $\mathrm{H}_{1}(X ; \mathbb{R})$. For the case of a linear flow $R^{\alpha}$ on $\mathbb{T}^{n}$, we have shown above that $\mathcal{S}\left(R_{t}^{\alpha}\right)=\{\alpha\} \subset$ $\mathbb{R}^{n} \equiv \mathrm{H}_{1}\left(\mathbb{T}^{n} ; \mathbb{R}\right)$.

The following corollary is an easy consequence of Proposition A.1.

Corollary A.3. For $i=1,2$, suppose that $\phi_{t}^{i}$ is a continous flow on the compact space $X_{i}$, which satisfies $\operatorname{Hom}\left(\left[X_{i}, \mathbb{T}\right], \mathbb{R}\right) \equiv \mathrm{H}_{1}\left(X_{i} ; \mathbb{R}\right)$. If $\psi: X_{1} \rightarrow X_{2}$ is a topological conjugacy between $\phi_{t}^{1}$ and $\phi_{t}^{2}$ (i.e. the map $\psi$ is a homeomorphism that satisfies $\psi \phi_{t}^{1}=\phi_{t}^{2} \psi$, for all $\left.t \in \mathbb{R}\right)$, then we have

$$
\mathcal{S}\left(\phi_{t}^{2}\right)=H_{1}(\psi)\left[\mathcal{S}\left(\phi_{t}^{1}\right)\right]
$$

We denote by $\mathfrak{F}(X)$ the set of continuous flows on $X$. We can embed $\mathfrak{F}(X)$ in $C^{0}(X \times[0,1], X)$ by the map $\phi_{t} \mapsto F^{\phi_{t}} \in C^{0}(X \times[0,1], X)$, where

$$
F^{\phi_{t}}(x, t)=\phi_{t}(x) .
$$

The topology on $C^{0}(X \times[0,1], X)$ is the compact open (or uniform) topology, and we endow $\mathfrak{F}(X)$ with the topology inherited from the embedding given above.

Lemma A.4. The map $\phi_{t} \mapsto \mathcal{S}\left(\phi_{t}\right)$ is upper semi-continuous on $\mathfrak{F}(X)$. This means that for each open subset $U \subseteq \mathrm{H}_{1}(X ; \mathbb{R})$, the set $\left\{\phi_{t} \in \mathfrak{F}(X) \mid \mathcal{S}\left(\phi_{t}\right) \subset U\right\}$ is open in $\mathfrak{F}(X)$.

Proof. Since the topology on $C^{0}(X \times[0,1], X)$ is metrizable, if this were not true we could find an open set $U \subset \mathrm{H}_{1}(X ; \mathbb{R})$ and a sequence $\phi_{t}^{n}$ of continuous flows on $X$ converging uniformly to a flow $\phi_{t}$, with $\mathcal{S}\left(\phi_{t}\right) \subset U$, and $\mathcal{S}\left(\phi_{t}^{n}\right)$ is not contained in $U$. This means that for each $n$ we can find a probability measure $\mu_{n}$ on $X$ invariant under $\phi_{t}^{n}$ and such that its Schwartzman asymptotic cycle $\mathcal{S}\left(\mu_{n}, \phi_{t}^{n}\right)$ for $\phi_{n}^{t}$ is 
not in the open set $U$. Since $\mathfrak{M}(X)$ is compact for the weak topology, extracting a subsequence if necessary, we can assume that $\mu_{n} \rightarrow \mu$. It is not difficult to show that $\mu$ is invariant under the flow $\phi_{t}$. We now show that $\mathcal{S}\left(\mu_{n}, \phi_{t}^{n}\right) \rightarrow \mathcal{S}\left(\mu, \phi_{t}\right)$. This will yield a contradiction and finish the proof because $\mathcal{S}\left(\mu_{n}, \phi_{t}^{n}\right)$ is in the closed set $\mathrm{H}_{1}(X ; \mathbb{R}) \backslash U$, for every $n$, and $\mathcal{S}\left(\mu, \phi_{t}\right) \in U$.

To show that the linear maps $\mathcal{S}\left(\mu_{n}, \phi_{t}^{n}\right) \in \mathrm{H}_{1}(X ; \mathbb{R})=\mathrm{H}^{1}(X ; \mathbb{R})^{*}$ converge to the linear map $\mathcal{S}\left(\mu, \phi_{t}\right)$, it suffices to show that $\mathcal{S}\left(\mu_{n}, \phi_{t}^{n}\right)([f]) \rightarrow \mathcal{S}\left(\mu, \phi_{t}\right)([f])$, for every $[f] \in[X, \mathbb{T}]=\mathrm{H}^{1}(X ; \mathbb{Z}) \subset \mathrm{H}^{1}(X ; \mathbb{R})=\mathrm{H}^{1}(X ; \mathbb{Z}) \otimes \mathbb{R}$. Fix now a continuous map $f: X \rightarrow \mathbb{T}$. Denote by $F_{n}, F: X \times[0,1] \rightarrow \mathbb{T}$ the maps defined by

$$
F_{n}(x, t)=f\left(\phi_{t}^{n}(x)\right)-f(x) \text { and } F(x, t)=f\left(\phi_{t}(x)\right)-f(x) .
$$

By the uniform continuity of $f$ on the compact metric space $X$, the sequence $F_{n}$ converges uniformly to $F$. Since $F_{n} \mid X \times\{0\} \equiv 0$, if we call $\tilde{F}_{n}: X \times[0,1] \rightarrow \mathbb{R}$ the lift of $F_{n}$ such that $\tilde{F}_{n} \mid X \times\{0\} \equiv 0$, then the sequence $\tilde{F}_{n}$ also converges uniformly to $\tilde{F}$, that is the lift of $F$ such that $\tilde{F} \mid X \times\{0\} \equiv 0$. Since the $\mu_{n}$ are probability measures, we have

$$
\left|\int_{X} \tilde{F}_{n}(x, 1) \mu_{n}(x)-\int_{X} \tilde{F}(x, 1) \mu_{n}(x)\right| \leq\left\|\tilde{F}_{n}-\tilde{F}\right\|_{\infty} \longrightarrow 0 .
$$

Since $\mu_{n} \rightarrow \mu$ weakly, we also have

$$
\left|\int_{X} \tilde{F}(x, 1) \mu_{n}(x)-\int_{X} \tilde{F}(x, 1) \mu(x)\right| \longrightarrow 0 .
$$

Therefore

$$
\mathcal{S}\left(\mu_{n}, \phi_{t}^{n}\right)([f])=\int_{X} \tilde{F}_{n}(x, 1) \mu_{n}(x) \rightarrow \mathcal{S}\left(\mu, \phi_{t}\right)([f])=\int_{X} \tilde{F}(x, 1) \mu_{0}(x) .
$$

Definition A.5 (Schwartzman unique ergodicity). We say that a flow $\phi_{t}$ is Schwartzman uniquely ergodic if $\mathcal{S}\left(\phi_{t}\right)$ is reduced to one point.

Theorem A.6. The set $\mathfrak{S}(N)$ of Schwartzman uniquely ergodic flows is a $G_{\delta}$-set in $\mathfrak{F}(X)$.

Proof. . Fix some norm on $\mathrm{H}^{1}(X ; \mathbb{R})$. We shall measure diameters of subsets of $\mathrm{H}^{1}(X ; \mathbb{R})$. with respect to that norm. Fix $\epsilon>0$. Call $\mathcal{U}_{\epsilon}$ the set of flows $\phi_{t}$ such that the diameter of $\mathcal{S}\left(\phi_{t}\right) \subset \mathrm{H}_{1}(X ; \mathbb{R})$ is $<\epsilon$. If $\phi_{t}^{0} \in \mathcal{U}_{\epsilon}$, we can find $U$ an open subset of $\mathrm{H}_{1}(X ; \mathbb{R})$ of diameter $<\epsilon$ and containing $\mathcal{S}\left(\phi_{t}^{0}\right)$. By the lemma above the set $\left\{\phi_{t} \in \mathfrak{F}(X) \mid \mathcal{S}\left(\phi_{t}\right) \subset U\right\}$ is open in $\mathfrak{F}(X)$ contains $\phi_{t}^{0}$ and is contained in $\mathcal{U}_{\epsilon}$. The set of Schwartzman uniquely ergodic flows is $\cap_{n \geq 1} \mathcal{U}_{1 / n}$.

Example A.7. By the computation done above, linear flows on the torus $\mathbb{T}^{n}$ are Schwartzman uniquely ergodic. Of course, all uniquely ergodic flows (i.e. flows having exactly one invariant probability measure) are also Schwartzman uniquely ergodic. Moreover, by Corollary A.3, any flow topologically conjugate to a Schwartzman uniquely ergodic flow is itself Schwartzman uniquely ergodic. 
Moreover, other examples can be obtained by the following result:

Proposition A.8. Let $\varphi_{t}: X \longrightarrow X$ be a continuous flow on the compact path connected space $X$. Suppose that there exist $t_{i} \uparrow+\infty$ such that $\varphi_{t_{i}} \longrightarrow \varphi$ in $C(X, X)$ (with the $C^{0}$-topology). Then, $\varphi_{t}$ is Schwartzman uniquely ergodic. In particular, periodic flows and (uniformly) recurrent flows are Schwartzman uniquely ergodic (in both cases $\varphi=\mathrm{Id}$ ).

Proof. Fix a continuous map $f: X \rightarrow \mathbb{T}$. Consider the function $F: X \times$ $[0,+\infty) \rightarrow \mathbb{T},(x, t) \mapsto f\left(\phi_{t}(x)\right)-f(x)$. We have $F(x, 0)=0$, for every $x \in X$. Call $\bar{F}: X \times[0,+\infty) \rightarrow \mathbb{R}$ the (unique) continuous lift of $F$ such that $\bar{F}(x, 0)=0$, for every $x \in X$. The definition of the Schwartzman asymptotic cycle gives

$$
\mathcal{S}(\mu)([f])=\int_{X} \bar{F}(x, 1) d \mu(x),
$$

for every probability measure invariant under $\phi_{t}$. We claim that we have

$$
\forall t, t^{\prime} \geq 0, \forall x \in X, \quad \bar{F}\left(x, t+t^{\prime}\right)=\bar{F}\left(\phi_{t}(x), t^{\prime}\right)+F(x, t) .
$$

In fact, if we fix $t$ and we consider each side of the equality above as a (continuous) function of $\left(x, t^{\prime}\right)$ with values in $\mathbb{R}$, we see that the two sides are equal for $t^{\prime}=0$, and that they both lift the function

$$
\left(x, t^{\prime}\right) \mapsto f\left(\phi_{t+t^{\prime}}(x)\right)-f(x)=f\left(\phi_{t}^{\prime}\left(\phi_{t}(x)\right)-f\left(\phi_{t}(x)\right)+f\left(\phi_{t}(x)\right)-f(x)\right.
$$

with values in $\mathbb{T}$. By induction, it follows easily that

$$
\forall k \in \mathbb{N}, \quad \bar{F}(x, k)=\sum_{j=0}^{k-1} \bar{F}\left(\phi_{j}(x), 1\right) .
$$

Therefore, if $t \geq 0$ and $[t]$ is its integer part, we also obtain

$$
\bar{F}(x, t)=\bar{F}\left(\phi_{[t]}(x), t-[t]\right)+\sum_{j=0}^{[t]-1} \bar{F}\left(\phi_{j}(x), 1\right) .
$$

It follows that

$$
\forall t \geq 0, \forall x \in X, \quad|\bar{F}(x, t)| \leq([t]+1)\|\bar{F} \mid X \times[0,1]\|_{\infty} .
$$

By compactness $\|\bar{F} \mid X \times[0,1]\|_{\infty}$ is finite. If we integrate equality $(*)$ with respect to a probability measure $\mu$ on $X$ invariant under the flow $\phi_{t}$, we obtain

$$
\int_{X} \bar{F}(x, t) d \mu(x)=\int_{X} \bar{F}(x, t-[t]) d \mu(x)+[t] \int_{X} \bar{F}(x, 1) d \mu(x) .
$$


Therefore we have

$$
\mathcal{S}(\mu)([f])=\lim _{t \rightarrow+\infty} \int_{X} \frac{\bar{F}(x, t)}{t} d \mu(x) .
$$

If $\gamma:[a, b] \rightarrow \mathbb{T}$ is a continuous path, with $a \leq b$, denote $\mathcal{V}(\gamma \mid[a, b]):=\bar{\gamma}(b)-$ $\bar{\gamma}(a)$, where $\bar{\gamma}:[a, b] \rightarrow \mathbb{R}$ is a continuous lift of $\gamma$ (this quantity does not depend on the chosen lift).

Suppose now that we set $\gamma_{x}(s)=\phi_{s}(x)$; we have $\bar{F}(x, t)=\mathcal{V}\left(f \gamma_{x} \mid[0, t]\right)$. Fix now some point $x_{0} \in X$, and consider $t_{i} \rightarrow+\infty$ such that $\phi_{t_{i}} \rightarrow \phi$ in the $C^{0}$ topology. Since $\bar{F}\left(x_{0}, t\right) / t$ is bounded in absolute value by $2\|\bar{F} \mid X \times[0,1]\|_{\infty}$, for $t \geq 1$, extracting a subsequence if necessary, we can assume that $\bar{F}\left(x_{0}, t_{i}\right) / t_{i} \rightarrow$ $c \in \mathbb{R}$. If $x \in X$, we can find a continuous path $\gamma:[0,1] \rightarrow M$ with $\gamma(0)=x_{0}$ and $\gamma(1)=x$. The map $\Gamma:[0,1] \times[0, t] \rightarrow \mathbb{T},\left(s, s^{\prime}\right) \mapsto \phi_{s^{\prime}}(\gamma(s))$ is continuous, therefore we can lift it to a continuous function with values in $\mathbb{R}$, and this implies the equality

$\mathcal{V}(\Gamma \mid[0,1] \times\{0\})+\mathcal{V}(\Gamma \mid\{1\} \times[0, t])-\mathcal{V}(\Gamma \mid[0,1] \times\{1\})-\mathcal{V}(\Gamma \mid\{0\} \times[0, t])=0$

This can be rewritten as

$$
\mathcal{V}\left(f \gamma_{x} \mid[0, t]\right)-\mathcal{V}\left(f \gamma_{x_{0}} \mid[0, t]\right)=\mathcal{V}\left(f \phi_{t} \gamma\right)-\mathcal{V}(f \gamma)
$$

which translates to

$$
\bar{F}(x, t)-\bar{F}\left(x_{0}, t\right)=\mathcal{V}\left(f \phi_{t} \gamma\right)-\mathcal{V}(f \gamma)
$$

Since $\phi_{t_{i}} \rightarrow \phi$ uniformly, by continuity of $\mathcal{V}$, the left hand-side remains bounded as $t=t_{i} \rightarrow+\infty$. It follows that $\left(\bar{F}\left(x, t_{i}\right)-\bar{F}\left(x_{0}, t_{i}\right)\right) / t_{i} \rightarrow 0$. Hence for every $x \in X$, we also have that $\bar{F}\left(x, t_{i}\right) / t_{i}$ tends to the same limit $c$ as $\bar{F}\left(x_{0}, t_{i}\right) / t_{i}$. Since $\bar{F}(x, t) / t$ is uniformly bounded for $t \geq 1$, by $(* *)$, by Lebesgue's dominated convergence we obtain from $(* * *)$ that $\mathcal{S}(\mu)([f])=c$, where $c$ is independent of the invariant measure $\mu$. This is of course true for any $f: X \rightarrow \mathbb{T}$. Therefore $\mathcal{S}(\mu)$ does not depend on the invariant measure $\mu$.

An interesting property of Schwartzman uniquely ergodic flows (which also shows that they have some kind of rigidity) is the following proposition, that follows immediately from the definition of Schwartzman unique ergodicity and what we remarked, in the examples above, about the asymptotic cycles of fixed and periodic points (see also [25]).

Proposition A.9. Suppose that $\phi_{t}$ is a Schwartzman uniquely ergodic flow on X. If there exists either a fixed point or a closed orbit homologous to zero, then all closed orbits are homologous to zero. In the remaining case, if $C_{1}$ and $C_{2}$ are closed orbits with periods $\tau_{1}$ and $\tau_{2}$, then $\frac{C_{1}}{\tau_{1}}$ and $\frac{C_{2}}{\tau_{2}}$ are homologous. Since $\left[C_{1}\right]$ and $\left[C_{2}\right]$ are in $\mathrm{H}_{1}(X ; \mathbb{Z})$, it follows in this case that the ratio of the periods of any two closed orbits must be rational. Consequently, for any continuous family of periodic orbits of $\varphi_{t}$, all orbits have the same period. 
Definition A.10 (Schwartzman strict ergodicity). We say that a flow $\phi_{t}$ is Schwartzman strictly ergodic if it is Schwartzman uniquely ergodic and it has an invariant measure $\mu$ of full support (i.e. $\mu(U)>0$ for every non-empty open subset $U$ of $X)$.

Example A.11. Linear flows on the torus $\mathbb{T}^{n}$ are Schwartzman strictly ergodic (they preserve Lebesgue measure). Of course, all strictly ergodic flows (i.e. flows having exactly one invariant probability measure, and the support of this measure is full) are also Schwartzman strictly ergodic. A minimal flow which is Schwartzman uniquely ergodic is in fact Schwartzman strictly ergodic (because all invariant measures have full support). Moreover, any flow topologically conjugate to a Schwartzman strictly ergodic flow is also Schwartzman strictly ergodic.

\section{References}

[1] V. I. ARNOL'D, Proof of a theorem of A. N. Kolmogorov on the preservation of conditionally periodic motions under a small perturbation of the Hamiltonian, Uspekhi Mat. Nauk 5 (113) 18 (1963), 13-40.

[2] A. Banyaga, "The Structure of Classical Diffeomorphism Groups", Mathematics and its Applications, Vol. 400, Kluwer Academic Publishers Group, Dordrecht, 1997.

[3] P. BERnARD, Existence of $C^{1,1}$ critical subsolutions of the Hamilton-Jacobi equation on compact manifolds, Ann. Sci. École Norm. Sup. (3) 40 (2007), 445-452.

[4] G. D. BIRKHOFF, Surface transformations and their dynamical applications, Acta Math. (1) 43 (1920); Sur quelques courbes fermées remarquables, Bull. Soc. Math. France (1) 60 (1932); reprinted in Collected Mathematical Papers, Vol. II, 111-418, respectively (New York: AMS 1950).

[5] H. Broer and F. TAKens, Unicity of KAM tori, Ergodic Theory Dynam. Systems (3) 27 (2007), 713-724.

[6] M. J. Dias CARNEIRo, On minimizing measures of the action of autonomous Lagrangians, Nonlinearity (6) 8 (1995), 1077-1085.

[7] O. Costin, G. Gallavotti, G. Gentile and A. Givliani, Borel summability and Lindstedt series, Comm. Math. Phys. (1) 269 (2007), 175-193.

[8] G. CONTRERAS and R. ITURRIAGA, Global minimizers of autonomous Lagrangians. In: " $22^{\circ}$ Colóquio Brasileiro de Matemática" [22nd Brazilian Mathematics Colloquium]. Instituto de Matemática Pura e Aplicada (IMPA), Rio de Janeiro, 1999, 148 pp.

[9] A. FATHI, Structure of the group of homeomorphisms preserving a good measure on a compact manifold, Ann. Sci. École Norm. Sup. (4) 13 (1980), 45-93.

[10] A. FATHI, "The Weak KAM Theorem in Lagrangian Dynamics", Cambridge University Press, to appear.

[11] A. FAthi and M. R. Herman, Existence de difféomorphismes minimaux, Astérisque 49 (1977), 37-59.

[12] A. FATHi and A. SiCONOLFI, Existence of $C^{1}$ critical subsolutions of the Hamilton-Jacobi equation, Invent. Math. (2) 155 (2004), 363-388.

[13] G. Gallavotti and G. Gentile , Hyperbolic low-dimensional invariant tori and summations of divergent series, Comm. Math. Phys. (3) 227 (2002), 421-460.

[14] G. Gentile and G. Gallavotti, Degenerate elliptic resonances, Comm. Math. Phys. (2) 257 (2005), 319-362.

[15] M. R. Herman, Inégalités "a priori" pour des tores lagrangiens invariants par des difféomorphismes symplectiques, Inst. Hautes Études Sci. Publ. Math. 70 (1989), 47-101. 
[16] M. R. HeRmAn, On the dynamics of Lagrangian tori invariant by symplectic diffeomorphisms, In: "Progress in variational methods in Hamiltonian systems and elliptic equations" (L'Aquila, 1990), Pitman Res. Notes Math. Ser., 243, Longman Sci. Tech., Harlow, 1992, 92-112.

[17] A. N. Kolmogorov, On conservation of conditionally periodic motions for a small change in Hamilton's function, Dokl. Akad. Nauk SSSR (N.S.) 98 (1954), 527-530.

[18] P.-L. Lions, G. Papanicolaou and S. R. SRinivasa Varadhan, Homogenization of Hamilton-Jacobi equation, Unpublished preprint, 1987.

[19] R. MAÑÉ, Lagrangian flows: the dynamics of globally minimizing orbits, Bol. Soc. Brasil. Mat. (N.S.) (2) 28 (1997), 141-153.

[20] J. N. MATHER, Action minimizing invariant measures for positive definite Lagrangian systems, Math. Z. (2) 207 (1991), 169-207.

[21] J. N. MATHER, Variational construction of connecting orbits, Ann. Inst. Fourier (Grenoble) (5) 43 (1993), 1349-1386.

[22] J. Moser, On invariant curves of area-preserving mappings of an annulus, Nachr. Akad. Wiss. Göttingen Math.-Phys. Kl. II, (1962), 1-20.

[23] D. SAlamon and E. Zehnder, KAM theory in configuration space, Comment. Math. Helv. (1) 64 (1989), 84-132.

[24] D. A. Salamon, The Kolmogorov-Arnold-Moser theorem, Math. Phys. Electron. J. 10: Paper 3, 37 pp. (electronic), 2004.

[25] S. Schwartzman, Asymptotic cycles, Ann. of Math. (2) 66 (1957), 270-284.

[26] A. Sorrentino, On the integrability of Tonelli Hamiltonians, preprint (2009), arXiV:0903.4300.

École Normale Supérieure de Lyon

Unité de Mathématiques Pures et Appliquées UMR CNRS 5669

46, allée d'Italie

69364 Lyon Cedex 07, France

Dipartimento di Matematica

Università degli Studi di Roma Tre

L.go S. Leonardo Murialdo 1

00146 Roma, Italia

Department of Mathematics

Princeton University

Fine Hall, Washington Road

Princeton NJ 08544-1000, USA

and

Department of Pure Mathematics

and Mathematical Statistics

University of Cambridge

Wiberforce Road

Cambridge CB3 OWB, UK 